\title{
Robotic Management of Fibroids: Discussion of Use, Criteria and Advantages
}

\author{
Ioannis D. Gkegkes ${ }^{1, *}$, George latrakis², Paraskevi-Evangelia lavazzo³, Konstadia Bakalianou4, \\ Christos lavazzo ${ }^{3}$
}

\section{ABSTRACT}

Fibroids are the most common benign tumors affecting fertility and quality of life. Different methods either definitive or fertility sparing are used for their management by using open, laparoscopic and robotic techniques. This is a narrative review presenting the role and the advantages of robotic surgery in fibroids (myomectomies or hysterectomies). Such a management is effective, safe and feasible in hands of well-trained teams even for multiple, large or deep located fibroids.

\section{KEYWORDS}

leiomyoma; fibroid; myomectomy; da Vinci@; robot; treatment

\section{AUTHOR AFFILIATIONS}

${ }^{1}$ Department of Surgery, Royal Devon and Exeter NHS Foundation Trust, Exeter, UK

2 Department of Obstetrics and Gynaecology, Technological Educational Institution of Athens, Athens, Greece

${ }^{3}$ Gynaecological Oncology Department, Metaxa Cancer Hospital, Piraeus, Greece

${ }^{4}$ Department of Obstetrics and Gynecology, General Hospital "Agia Olga", Athens, Greece

* Corresponding author: 11 Tuckfield House, Residential Village, EX2 5DS, Bovemoors Lane, Exeter, UK; e-mail: ioannisgkegkes@gmail.com 


\section{INTRODUCTION}

Fibroids are the most common benign uterine tumors deriving from the smooth muscle cells of the myometrium. They can cause uterine bleeding, pain, pressure symptoms and infertility (1). Depending on their anatomic location they are characterised as subserosal, intramural, submucosal and pedunculated fibroids. Ultrasound is the most widely used modality for fibroids diagnosis due to its availability and cost-effectiveness. Magnetic resonance imaging (MRI) is the best modality for visualizing the size and location of all uterine myomas and rule out adenomyosis. However, due to the expense of MRI, its use is best reserved for surgical planning for complicated procedures (2). Initially, expectant management could be a reasonable option for some women with fibroids or, "empirically", fibroids are treated conservatively either with progestin-only treatments or combined hormonal contraceptives or GnRH analogues (3). However, they are the primary indication of hysterectomies in order to have a definitive treatment. Their management could also be fertility sparing if that is possible by performing myomectomy or uterine embolization (1). Laparoscopic or open techniques, depending on each surgeon's preference and experience, are offered as treatment options while recently robotic procedures are also suggested.

The da Vinci ${ }^{\oplus}$ surgical system (Intuitive Surgical, Sunnyvale, California) received FDA approval in 2005. Robotic procedures have been introduced in order to improve surgical performance. Increased dexterity, greater range of motion and better depth perception are the main advantages of robotic-assisted techniques. Its limitations include lack of tactile feedback and increased cost. Robotic procedures combine the advantages of open and laparoscopic procedures and are another alternative in the management of fibroids either with myomectomy or with hysterectomy (4).

The aim of this narrative review is to present the use, the criteria and the advantages of robotic surgery in the treatment of fibroids.

\section{DISCUSSION}

Different techniques including open, laparoscopic or robotic procedures are "concurrently" used in the management of fibroids and robotic-assisted procedures are becoming more common (4). The patient is preoperatively assessed with imaging scans including ultrasound and MRI and she signs the informed consent when she is informed about the type of planned procedure and possible risks of it including infection, bleeding and injury of adjacent organs. "A little arbitrarily", candidates for robotic myomectomy are all the patients with any single myoma $<15 \mathrm{~cm}$ or with $<15$ myomas in total while palpation of the uterine fundus above the umbilicus, with diffuse adenomyosis or a uterine cavity which cannot be clearly visualized by imaging techniques are contraindications of robotic myomectomy (5). In these cases, a total or subtotal hysterectomy could be performed.

Patient is operated in dorsal lithotomy position in Allen stirrups with the arms padded and tucked. After trocar placement, the patient would lie in Trendelenburg position. The assistant's laparoscopic port is used for suction/ irrigation, passage of needles, tissue retraction, and morcellation. The most commonly used robotic instruments are Cadiere or Maryland bipolar forceps, harmonic shears, and mega or large needle driver. Initially, the fibroid location is exactly determined and then vasopressin is injected into the myometrium surrounding the fibroid. An incision is performed over the fibroid in a longitudinal or horizontal axis followed by enucleation of it by using a robotic tenaculum and/or a bipolar coagulator in addition to the harmonic shears. The assistant could also help by additional traction with a laparoscopic tenaculum. Multilayer closure employing barbed sutures is usually performed. The specimen is morcelated by taking care of the adjacent tissues especially the bowel and the specimen is retrieved through the assistant's port.

Regarding the surgical steps of a robotic hysterectomy, two Vicryl ${ }^{\odot}$ sutures are put on the cervix and then the uterus is instrumented with manipulator and a cervical cap is tied with the sutures. An indwelling foley catheter is also used at outset. Then, uncomplicated Veress needle is fitted and pneumoperitoneum and trocars insertion are performed followed by side docking and instrumentation. Usually, the bipolar diathermy (DT) is set at 40 and the scissors are put through the right main port with monopolar DT at 40 cut and coagulation. Cadiere or Maryland forceps are placed in the third arm. Then, after incising with monopolar DT, the broad ligaments are entered and the ureters are identified bilaterally. The infundibulopelvic pedicles and round ligaments are "taken" with bipolar and/ or monopolar DT. The uterovesical fold is taken with monopolar DT and reflection of the bladder follows. The uterine vessels are then skeletalised and taken with bipolar and monopolar DT. The vagina is entered anteriorly on top of the manipulator's cervical cap and the dissection is continued circumferentially using monopolar DT. The uterine specimen could be extracted through the vagina, through a mini-laparotomy or morcellated depending on its dimensions and suspicion for malignancy. The vaginal vault is then closed with continuous barbed suture. Regarding the postoperative care, the patients are early mobilised and usually are discharged the first postoperative day. In future pregnancies, the risk of uterine rupture is very low when the myometrium is appropriately repaired (6).

Robotic system preserves the advantages of conventional laparoscopy while it offers the possibility to the gynaecologist to operate with more dexterity on the uterus, especially when performing a myomectomy. The articulated instruments permit a wide range of motions while they increase the ability of the surgeon to work efficiently. The 3 -dimention stereoscopic vision by the use of binocular optics, the filtration of the tremor and the less operator fatigue are some of the obvious advantages of such operations. It is suggested that a diagnostic laparoscopy should be used in order to clarify the exact positions of the trocars in order to have uncomplicated access to the pelvis. Robotic procedures can be safely performed after taking into account the physiological changes of pneumoperitoneum and steep Trendelenburg position during a preoperative anaesthetic review (7). The $\mathrm{CO}_{2}$ pressure required 
for exposure is often lower in correlation with traditional laparoscopy as result of the mechanical lift of the robot (8). All the above mentioned advantages can lead to more anatomical procedures.

A recent meta-analysis revealed that robotic procedures have significant short term benefits compared to open surgery but the results were found to be similar to laparoscopic procedures (9). It was shown that there is no significant difference in number and weight of fibroids or operating times when comparing robotic with laparoscopic or open myomectomy $(10,11)$. However, blood loss is less in the robotic group (10). When comparing robotic with open myomectomies, although the operative time is double (261 versus 125 minutes), the hospital stay was half the days in the robotic cases (1.5 versus 2.7 days) (12). In all those studies, the rates of conversion to open surgery and blood transfusion are minimal. The most common reason of conversion is the location or the volume of the fibroid and/or the luck of experience of the surgeon. Regarding the pregnancy outcomes after robotic myomectomies, a cohort study revealed that the mean time to conception was $12.9 \pm 11.5$ months, spontaneous abortions occurred in $18.9 \%$, while preterm delivery in $17.4 \%$ of the achieved pregnancies. The uterine rupture rates were $1.1 \%$ (13). Another study showed that the pregnancy rate is $69 \%$ and the natural conception rate $55 \%$ after robotic myomectomies (14). Additionally, preoperative obesity is not a contraindication or risk factor of poor outcome for women undergoing robotic myomectomy (15). So, the robotic assisted surgery also permits the realization of a key hole operation (at most 10 to $12 \mathrm{~mm}$ ) (8) which can be interpreted into significantly less blood loss, less pain, shorter recovery time as well as shorter hospital stay, quicker return to normal activity and better aesthetic result. Moreover, the learning curve of such operations is rapid compared to laparoscopy, while at the same time the good clinical outcomes are equally effective as conventional laparoscopy and within similar operating times.

On the other hand, the most significant disadvantages include the high costs of use, the bulky machinery and the need for staff training. Regarding the cost, it was shown that open myomectomy costs $\$ 4,937$ compared to laparoscopy which costs $\$ 6,219$ and robotic procedures $\$ 7,299$ (16). Of course, entry of new robotic systems in the market as well as use of the robot by different surgical teams and in a high volume of patients could decrease the cost disadvantage. Moreover, some authors argue that robotic are longer operations and the loss of tactile sensation makes difficult the sensation of an intramural fibroid (17).

We present a state of the art narrative review dealing with management of uterine fibroids, especially the surgical treatment using the da Vinci ${ }^{\oplus}$ robotic system. We briefly describe the fertility sparing procedure - myomectomy - and the definitive procedure - hysterectomy - pointing out the advantages of the robotic surgery (increased dexterity, maneuverability of the system, greater range of motion and better depth perception) and compare the robotic, laparoscopic and open surgery in terms of complication rate, operative time, hospital stay, blood loss, learning curve and cost.

The review mentioned both approaches conservative and definitive with bigger focus on conservative approach.
We include a comparison of the laparotomic versus laparoscopic versus robotic assisted surgery. Recent meta-analyses and prospective studies are favouring the later two for shorter hospital stay and less blood loss (18-20). We would also like to note that morcelation of the tissue of fibroid after myomectomy - especially use of power morcellation could rise a controversy according to FDA advise $(21,22)$. For this reason, we suggest that any type of morcellation should be performed inside a laparoscopic bag to avoid tissue contamination (22).

Robotic system in conservative management is a very interesting modality, though it struggles in comparison with laparoscopic management because it brings hardly any advantage for its higher cost. There are still lot of countries where the myomectomy with robotic system is still not covered by insurance companies and the spending power of patients is still very low and this modality is therefore unavailable. But this is the controversy worth to mention. When the cost issue is going to get overcome that could be the time when robotic modality in conservative management of fibroids would become more common in the future.

\section{CONCLUSION}

In conclusion, robotic myomectomy or hysterectomy is an equally effective, feasible and safe alternative in welltrained hands compared to the traditional methods of open or laparoscopic surgery. The till now evidence shows that robotic myomectomy has comparable results to open and laparoscopic techniques. More randomized prospective studies are necessary to clarify the role of robotic management especially in the long term outcomes such as pregnancy, miscarriage, caesarean section and live birth rates.

\section{CONFLICT OF INTEREST}

No conflict of interest was declared by the authors.

\section{FINANCIAL DISCLOSURE}

The authors declared that this study has received no financial support.

\section{REFERENCES}

1. Falcone T, Parker WH. Surgical management of leiomyomas for fertility or uterine preservation. Obstet Gynecol 2013; 121: 856-68.

2. Kim M, Kim MK, Kim ML, et al. Robotic single-site myomectomy: A single-center experience of 101 consecutive cases. Int J Med Robot 2019 Sep 19: e1959.

3. Stewart EA, Lytle BL, Thomas L, et al. The Comparing Options for Management: PAtient-centered REsults for Uterine Fibroids (COMPARE-UF) registry: rationale and design. Am J Obstet Gynecol 2018; 219: 95.e1-95.

4. Iavazzo C, Gkegkes ID. Application of da Vinci ${ }^{\circledR}$ Robot in simple or radical hysterectomy: Tips and tricks. J Turk Ger Gynecol Assoc 2016; 17: 106-9.

5. Quaas AM, Einarsson JI, Srouji S, Gargiulo AR. Robotic myomectomy: a review of indications and techniques. Rev Obstet Gynecol 2010; 3: $185-91$. 
6. Mahajan N, Moretti ML, Lakhi NA. Spontaneous early first and second trimester uterine rupture following robotic-assisted myomectomy. J Obstet Gynaecol 2019; 39: 278-80.

7. Pathirana S, Kam P. Anaesthetic issues in robotic-assisted minimally invasive surgery. Anaesth Intensive Care 2018; 46: 25-35.

8. Iavazzo C, Gkegkes ID. Port site metastases after robot-assisted surgery: a systematic review. Int J Med Robot 2013; 9: 423-7.

9. Wang T, Tang H, Xie Z, Deng S. Robotic-assisted vs. laparoscopic and abdominal myomectomy for treatment of uterine fibroids: a meta-analysis. Minim Invasive Ther Allied Technol 2018; 27: 249-64.

10. Pluchino N, Litta P, Freschi L, et al. Comparison of the initial surgical experience with robotic and laparoscopic myomectomy. Int J Med Robot 2014; 10: 208-12.

11. Gobern JM, Rosemeyer CJ, Barter JF, Steren AJ. Comparison of robotic, laparoscopic and abdominal myomectomy in a community hospital. JSLS 2013; 17: 116-20.

12. Griffin L, Feinglass J, Garrett A, et al. Postoperative outcomes after robotic versus abdominal myomectomy. JSLS 2013; 17: 407-13.

13. Pitter MC, Gargiulo AR, Bonaventura LM, Lehman JS, Srouji SS. Preg nancy outcomes following robot assisted myomectomy. Hum Reprod 2013; 28: 99-108.

14. Lonnerfors C, Persson J. Pregnancy following robot-assisted laparoscopic myomectomy in women with deep intramural myomas. Acta Obstet Gynecol Scand 2011; 90(9): 972-7.
15. George A, Eisenstein D, Wegienka G. Analysis of the impact of body mass index on the surgical outcomes after robot-assisted laparoscopic myomectomy. J Minim Invasive Gynecol 2009; 16: 730-3.

16. Behera MA, Likes CE, Judd JP, Barnett JC, Havrilesky LJ, Wu JM. Cost analysis of abdominal, laparoscopic and robotic assisted myomectomies. J Minim Invasive Gynecol 2012; 19: 52-7.

17. Alkatout I, Mettler L, Günther V, et al. Safety and economical innovations regarding surgical treatment of fibroids. Minim Invasive Ther Allied Technol 2016; 25: 301-13.

18. Iavazzo C, Mamais I, Gkegkes ID. Robotic assisted vs laparoscopic and/or open myomectomy: systematic review and meta-analysis of the clinical evidence. Arch Gynecol Obstet 2016; 294: 5-17.

19. Wang T, Tang H, Xie Z, Deng S. Robotic-assisted vs. laparoscopic and abdominal myomectomy for treatment of uterine fibroids: a meta-analysis. Minim Invasive Ther Allied Technol 2018; 27: 249-64.

20. Pundir J, Pundir V, Walavalkar R, Omanwa K, Lancaster G, Kayani S. Robotic-assisted laparoscopic vs abdominal and laparoscopic myomectomy: systematic review and meta-analysis. J Minim Invasive Gynecol 2013; 20: 335-45.

21. Wallis L. FDA warns against power morcellation for hysterectomy and fibroids. Am J Nurs 2014; 114: 16.

22. Glaser LM, Friedman J, Tsai S, Chaudhari A, Milad M. Laparoscopic myomectomy and morcellation: A review of techniques, outcomes, and practice guidelines. Best Pract Res Clin Obstet Gynaecol 2018; 46: 99-112. 